\title{
Le volume TARMED par patient reste stable depuis des années
}

\author{
Kerstin Schutz, Sabine Zehnder
}

Expertes, division Médecine et tarifs ambulatoires

1 Ce calcul englobe toutes les disciplines médicales, les lois LAMal, LAA, LAI, LAM et tous les tarifs découlant de ces lois. 2 Pandémie de COVID-19: coûts supplémentaires de 735 millions. Oeschger C, Prantl A. Bull Med Suisses 2020;101(44):1442-4.

3 Legroupe de spécialités médicales «invasives» comprend les disciplines suivantes: chirurgie, chirurgie de la main, chirurgie plastique, gynécologie et obstétrique, ophtalmologie, orthopédie, oto-rhino-laryngologie, urologie.

4 Legroupe des médecins «de premier recours" comprend les disciplines suivantes: médecine interne générale, pédiatrie ainsi que les médecins praticiens.

5 Legroupe des médecins «spécialistes» comprend les disciplines suivantes: angiologie, cardiologie, dermato logie, endocrinologie, gastroentérologie, hématologie, infectiologie, médecine physique, néphrologie, neurologie, oncologie rhumatologie.

Grâce à la collecte de données effectuée par les médecins, toutes les données de facturation concernant l'année 2020 sont maintenant disponibles et peuvent être analysées. Si l'on considère le volume TARMED par cabinet médical, le bilan annuel 2020 se solde par une perte, en comparaison de celui de l'année 2019. Pour certaines disciplines médicales, les effets de l'interdiction de traitement et du semi-confinement ont pu être partiellement compensés par un travail supplémentaire considérable, accompli durant la seconde partie de l’année. médicale, pneumologie,

En avril passé, la situation de la pandémie de COVID-19 ne semblait offrir que de sombres perspectives. Les cabinets médicaux accusaient une perte de leur volume de facturation TARMED allant jusqu'à 40\% par rapport à l'année précédente pour certaines disciplines, de même qu'une diminution équivalente des contacts avec les patients (fig. 1). Dans l'ensemble, le chiffre d'affaires total ${ }^{1}$ par cabinet médical a diminué d'environ $14 \%$ durant le deuxième trimestre. En outre, bien que tout à fait justifiés, les coûts supplémentaires ${ }^{2}$ engendrés par les mesures de protection accrues ont cependant placé le corps médical devant des défis majeurs. Les mesures édictées ont eu un impact différent selon la discipline considérée: dans les disciplines invasives ${ }^{3}$ par exemple, près de la moitié des traitements n'ont pas pu être réalisés durant la période de restrictions. Toutefois, il a été possible de rattraper certaines inter-

\section{Comparaison des chiffres clés de 2020}

(par rapport à 2019)

\section{$-1.87 \%$}

Volume TARMED par consultation ventions par la suite grâce à un important travail supplémentaire et de compenser ainsi quelque peu la perte subie durant le printemps. Pour les disciplines invasives, le volume TARMED a diminué en moyenne de 3,12\% sur l'ensemble de l'année 2020.

\section{L'abondance après la pénurie}

Dans les cabinets médicaux de premier recours ${ }^{4}$, comme dans les cabinets des domaines spécialisés ${ }^{5}$, les patients qui n'ont pas nécessité une prise en charge urgente pendant la période de restriction de traitement ont simplement ajourné leurs demandes de prestations médicales jusqu'à la levée des restrictions. C'est ainsi qu'en juin 2020, par exemple, le volume TARMED par cabinet médical était de $20 \%$ supérieur à celui de l'année précédente (fig. 2).

Par ailleurs, la demande en prestations psychiatriques a globalement augmenté en 2020. Cette progression est vraie tant pour les patients déjà suivis que pour les nouveaux patients, avec également un nombre de premières consultations un peu plus élevé, même durant la période de semi-confinement en mars et avril. Cela explique la légère croissance du volume TARMED par rapport à l'année précédente (fig. 2).

Pour l'ensemble des disciplines médicales, l'année 2020 se termine avec une perte moyenne de 1,15\% du volume TARMED par cabinet médical (fig. 1). On observe cependant des différences d'une discipline à l'autre. Par exemple, la pédiatrie, l'oto-rhino-laryngologie, la pneumologie et la néphrologie affichent une perte supérieure à $8 \%$, contre une hausse d'environ 3 à $4 \%$ pour la rhumatologie, la psychiatrie et la radiologie. 


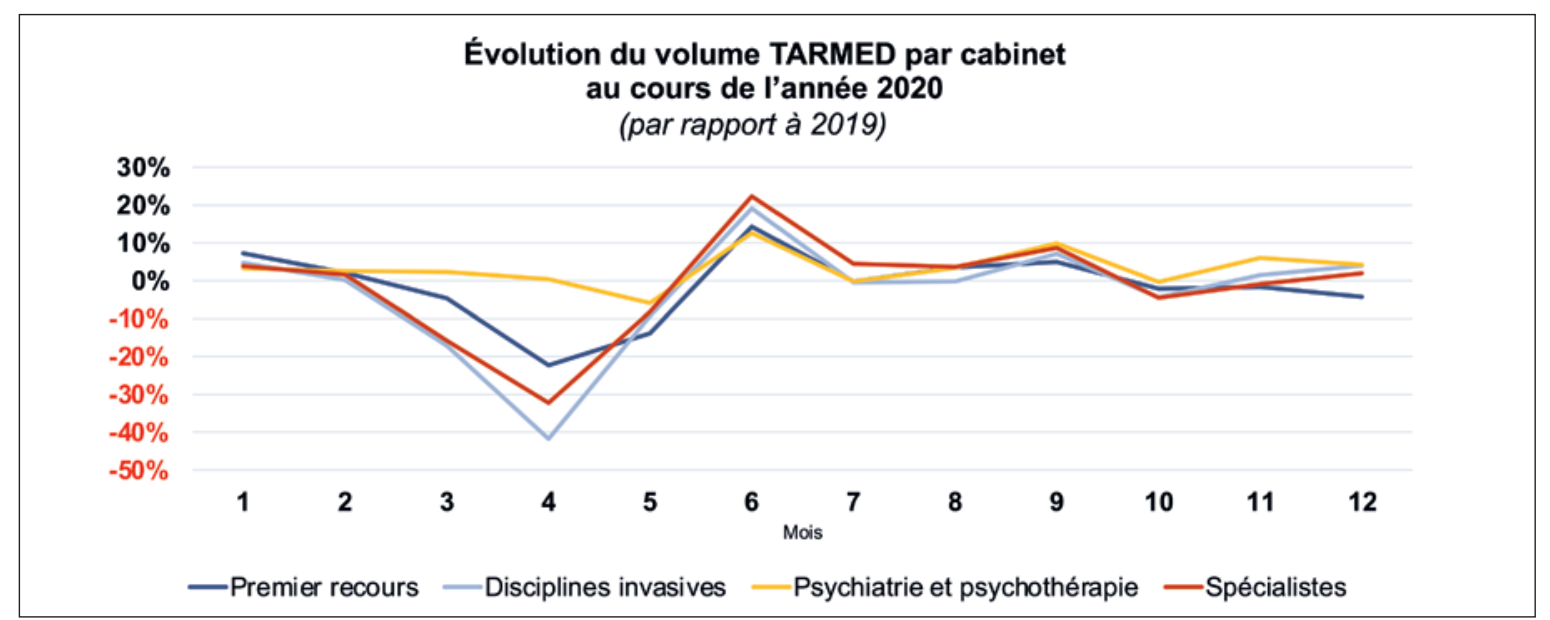

Figure 2: Evolution du volume TARMED (points tarifaires) par cabinet médical, au cours des mois de l'année 2020 (par rapport à I'année précédente); en fonction de différents groupes de spécialités; LAMal; Suisse entière; sans extrapolation.

\section{Moins de premiers contacts}

Le volume TARMED moyen par consultation a baissé de près de $2 \%$ (fig. 1), ce qui peut être attribué à la conversion de consultations ordinaires en prestations de télémédecine, en particulier pendant les mois de mars à mai. En

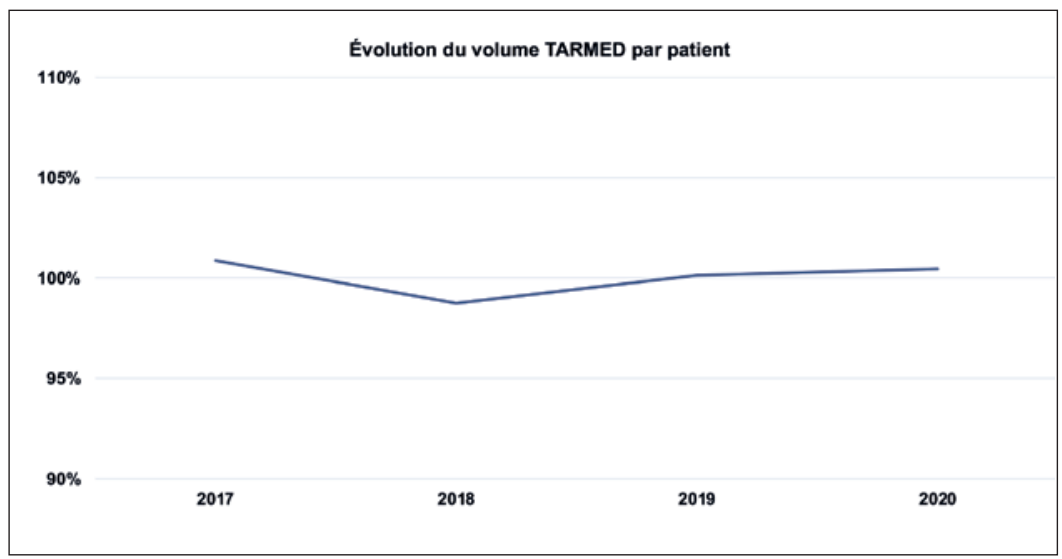

Figure 3: Evolution du volume TARMED (points tarifaires) par patient; toutes disciplines médicales confondues; LAMal; Suisse entière; sans extrapolation.

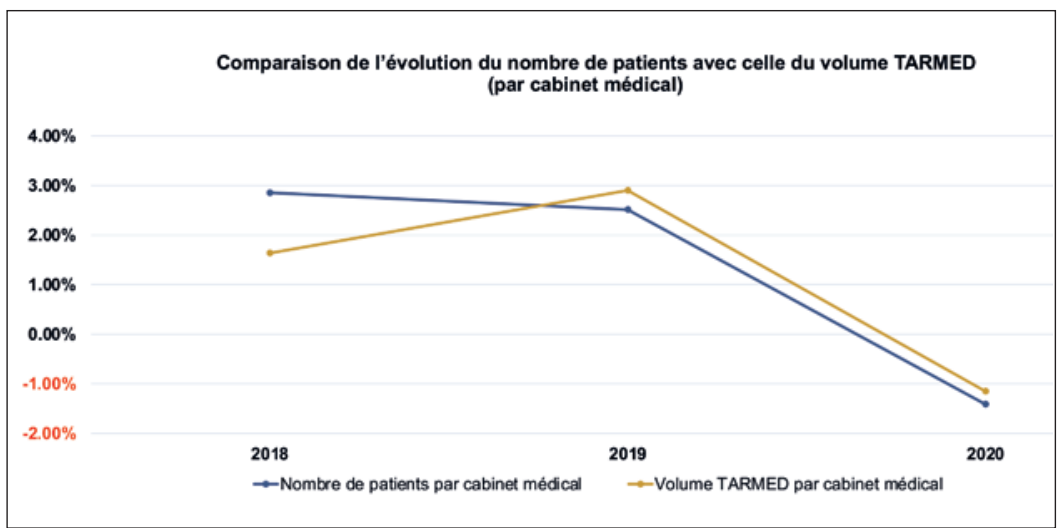

Figure 4: Evolution du nombre de patients par cabinet médical et évolution du volume TARMED (points tarifaires) par cabinet médical, toutes disciplines médicales confondues; LAMal; Suisse entière; sans extrapolation.
2020, le nombre de premières consultations par cabinet médical (patients par cabinet) a diminué de près de 1,4\% (fig. 1), contrairement à la légère hausse habituelle.

En outre, sur l'année 2020, le volume TARMED moyen par patient est resté stable (fig. 3). Cela signifie que les patients sollicitent des prestations médicales indépendamment de la situation de pandémie.

Le volume TARMED total par patient est stable depuis 2017, voire en légère baisse. Cela signifie également que l'intervention tarifaire de 2018 n'a pas mené à un accroissement compensatoire du volume TARMED. En effet, l'augmentation du volume total découle de l'augmentation du nombre de premiers contacts.

La figure 4 illustre le même phénomène d'un point de vue différent: le volume TARMED par cabinet médical évolue de manière proportionnelle au nombre de patients par cabinet. L'augmentation ou la diminution des coûts est directement liée au nombre de patients, dont la demande ne peut être contrôlée par le corps médical que dans une mesure très limitée.

\section{Davantage de télémédecine}

La conversion des consultations ordinaires en prestations de télémédecine a pu être observée tout au long de l'année 2020. Cette évolution concerne, à divers degrés, toutes les disciplines médicales. Si cette transition s'avère importante, notamment dans le domaine de la psychiatrie, elle n'est possible que pour certaines prestations particulières dans d'autres disciplines spécialisées. La figure 5 montre clairement qu'au cours des mois de mars à mai 2020, les consultations de télémédecine ne sont pas ajoutées aux consultations classiques, mais qu'elles ont plutôt partiellement compensé les consultations qui n'étaient plus possibles en présence physique. 


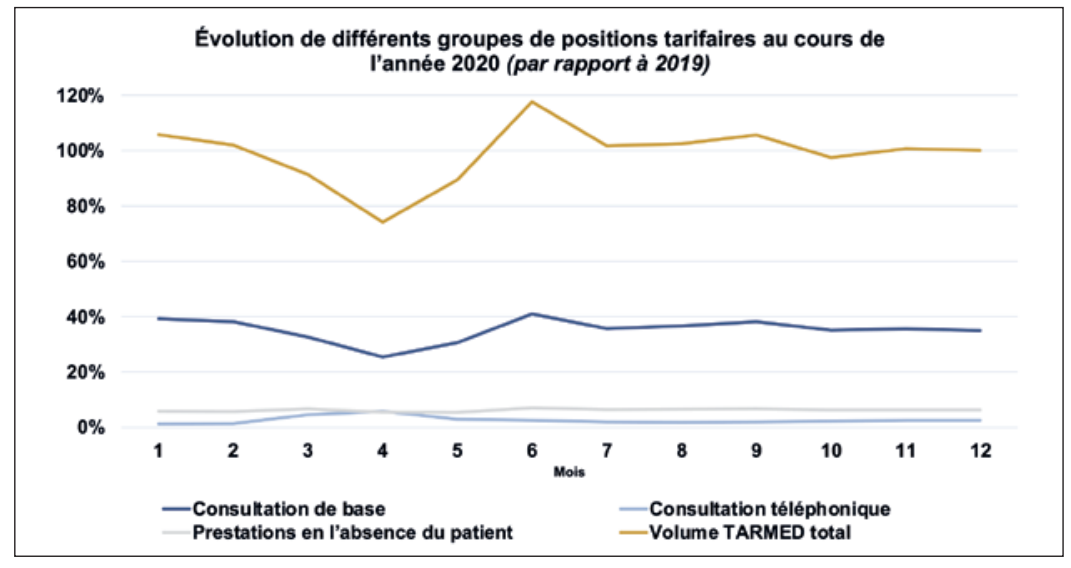

Figure 5: Evolution du volume TARMED (points tarifaires), au cours des mois de I'année 2020 (par rapport à l'année précédente), en fonction de différents groupes de positions tarifaires; toutes disciplines médicales confondues; LAMal; Suisse entière; sans extrapolation.

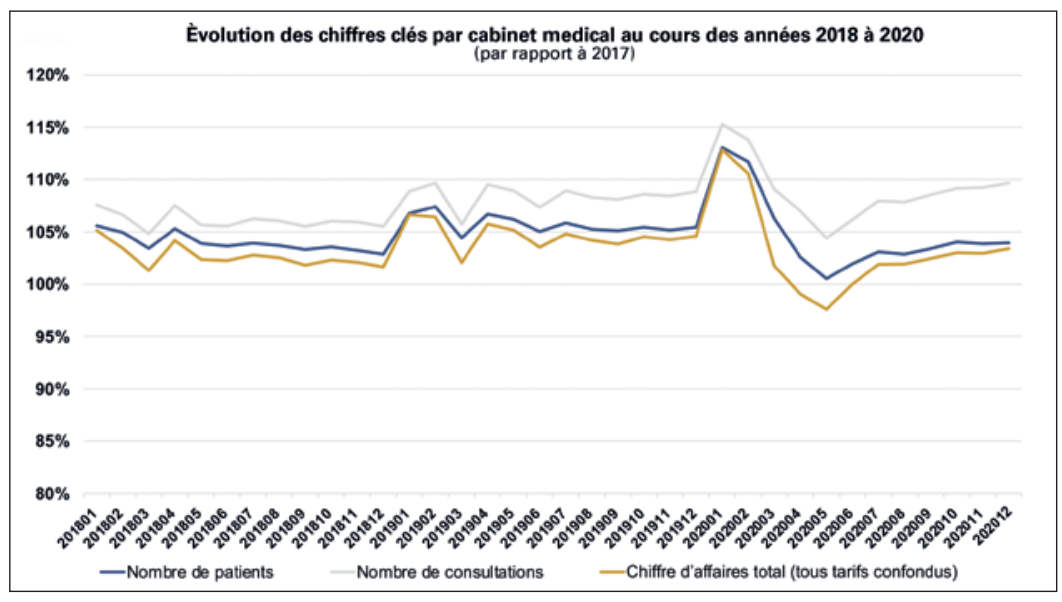

Figure 6: Evolution des chiffres clés, au cours des mois de 2018 à 2020

(par rapport à l'année 2017); toutes disciplines médicales confondues; LAMal; Suisse entière; sans extrapolation.

6 La livraison des données a lieu conformément au Code NAKO (centre de consolidation national). Le Codex NAKO réglemente l'accès et l'exploitation des données par des associations et des tiers. Elle porte sur les données agrégées représentant le chiffre d'affaires d'un cabinet médical moyen.

\section{FMH}

Division Médecine

et tarifs ambulatoires

Baslerstrasse 47

CH-4600 Olten

Tél. 0313591230

Fax 0313591238

tarife.ambulant[at]fmh.ch
Ces dernières années, le travail en l'absence du patient n'a cessé d'augmenter, et il n'est pas étonnant que cette tendance caractérise également l'année 2020, marquée par la pandémie de COVID-19. Les prestations facturées en l'absence du patient augmentent toutefois de manière modérée, passant d'une moyenne de 6,16 à 6,87 points tarifaires par consultation. Sur un total de 108,67 points par consultation (toutes disciplines médicales confondues), la part moyenne des prestations en l'absence du patient est légèrement supérieure à $6 \%$. L'augmentation significative du nombre de consultations par cabinet médical durant les mesures de restriction (fig. 6) peut également s'expliquer par l'augmentation des consultations téléphoniques, généralement plus brèves que les consultations ordinaires en présence physique. L'échange fréquent entre médecin et patient s'avère particulièrement nécessaire en cas de comorbidités. Chez des patients qui présentent des maladies chroniques ou multiples, la crainte de l'infection par le COVID-19 joue aussi probablement un rôle; plus l'insécurité est grande, plus ces patients contactent fréquemment leur médecin par téléphone.

\section{De nombreux traitements suspendus ont pu être rattrapés}

L'analyse du volume TARMED total et d'autres chiffres clés qui lui sont liés, tels que le nombre de patients (fig. 6), reflète à nouveau clairement la situation de pandémie: après une forte réduction durant les mois de printemps (durant lesquels le chiffre d'affaires par cabinet médical s'est situé bien en dessous des valeurs de 2017), de nombreux cabinets médicaux ont par la suite pu rattraper les traitements suspendus, moyennant un effort considérable. Cependant, pour presque toutes les disciplines médicales, on peut observer une baisse du volume total, le niveau de l'année précédente n'étant pas atteint.

Lévolution du volume TARMED par patient s'est montrée constante au cours de l'année 2020, par rapport aux années précédentes. Cela signifie qu'au cours des mois d'été et d'automne, seuls les traitements et interventions qui n'ont pas pu être réalisés au printemps ont été rattrapés.

\section{Les données relevées par les médecins \\ permettent des analyses rigoureuses}

Les données collectées par les médecins permettent d'analyser de manière détaillée l'évolution des données de facturation des cabinets médicaux. Ces dernières années, pas moins de 10000 d'entre eux ont livré activement leurs données et financé cette collecte par leurs cotisations aux centres de confiance cantonaux. Vu que la Société Vaudoise de Médecine (SVM) et des parties de Suisse centrale poursuivent leurs propres solutions, nous atteignons une couverture de près de $65 \%$ sur l'ensemble de la Suisse. Le pool de données du corps médical suisse comprend actuellement 316 millions de factures (copies), soit près de 3,2 milliards de fichiers et de données de prestations (état: fin mars 2021). De cette manière, les données peuvent être analysées selon les indications disponibles sur chaque facture. En outre, l'analyse permet de déterminer quelles prestations sont fréquemment fournies conjointement lors d'une consultation ou quelles positions tarifaires constituent une consultation moyenne.

Ces analyses complètes sont uniquement possibles grâce à tous les cabinets médicaux qui transmettent leurs données de facturation aux centres de confiance cantonaux. Leur nombre ne cesse de s'accroître et nous remercions vivement tous les médecins qui fournissent leurs données, car ils alimentent notre réservoir de données, le cofinancent et contribuent ainsi à ce que la FMH puisse fournir des analyses précises de l'activité du corps médical. Depuis début 2021, le Secrétariat d'Etat à l'économie (SECO) collabore avec la FMH; dorénavant, la FMH fournira au SECO des données agrégées sur les chiffres d'affaires concernant la Suisse entière $^{6}$. Le SECO utilisera ces données dans le but de surveiller la situation économique dans le secteur de la santé. Cette collaboration, qui souligne l'importance de la collecte des données effectuée par les médecins, nous réjouit particulièrement. 